\title{
A Fine-Grained Data Access Control using Attribute-Based Keyword in Web Search
}

\author{
P. Periyasamy ${ }^{1}$, K.Y. Pradheep ${ }^{2}$, U. Santhosh Kumar ${ }^{3}$, G. Vengadesan ${ }^{4}$ \\ UG, Department of CSE, M. Kumarasamy College of Engineering, Karur, India ${ }^{1,2,3,4}$
}

\begin{abstract}
To hold those critical user data secured from untrusting servers, present ideas generally use cryptographic techniques by revealing data decryption keys simply to accredited users. These answers unavoidably introduce key distribution and data management on a heavy calculation on the data owner when fine grained data access control is preferred. It does not scale well. The issue still remains unresolved for rapidly attaining fine-graininess scalability and data privacy of access control. Users outsource critical data for distribution on cloud hence, they projected some services for data safety and access control. Existing paper generally focuses on outsourced data-set that can be retrieved from various owners. It can be identifiable by several users.It uses the techniques of attribute based encryption called Attribute-based keyword search pattern with efficient user cancellation (ABKS-UR). Users can have own search capabilities by not trusting on an online related authority. It enables scalable fine-grained search approval.Our proposed scheme enables application scenarios which enables separate data file to be related with a set of appearances. We achieve this objective by manipulating and individually joining the techniques of Key Policy attribute based encryption (KP-ABE) and Flap encryption. This also has salient features of Users secret key responsibility thereby achieving finegraininess, scalability and data privacy for data access control. This examination shows that our proposed pattern is extremely efficient and provable safeguarding under existing security replicas.
\end{abstract}

Keywords: Cloud Computing, Management Data, Data Security.

\section{INTRODUCTION}

Cloud Computing is a recently emerged Computing terminology. In this real world the Cloud Computing, has become a feasible solution for sharing Computing possessions. Cloud Computing comprises clusters of isolated servers and software grids that agree data database and internet access to devices. Clouds can be separated as free, isolated or hybrid.

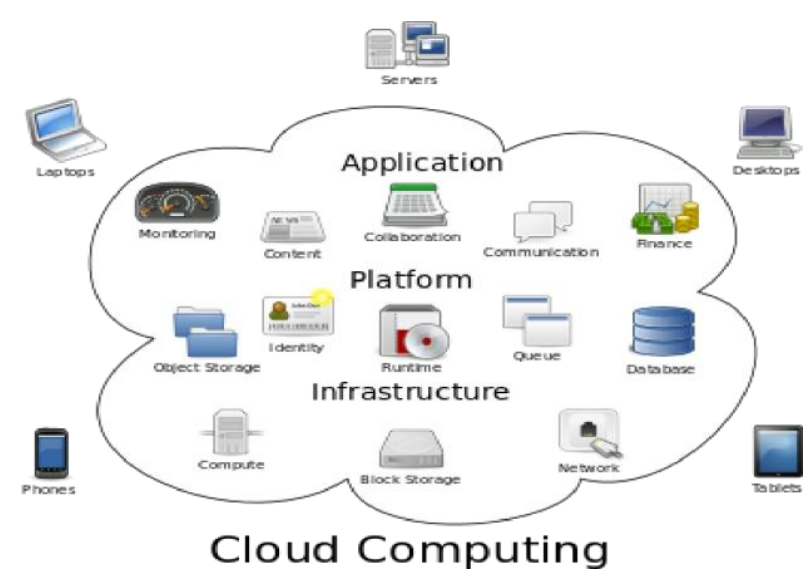

Cloud Computing based on distribution of services to attain logical and financial prudence of scale, like to an efficacy concluded a net. At the base of unrestricted Cloud Computing stays the concept of organized structure and distributed resources. Cloud Computing, or in simpler just "the Cloud", which too efforts on increasing the usefulness of the distributed services. Cloud allotting different services to multiple handlers. For example, a Cloud computer capability that provides European operators during European working hours with a unique application (e.g., email) which may change the same properties to provide North American operators during North America's working hours with a specific application (e.g., a web server).

This concept should increase the use of systems thus falling ecological damage as it is fewer power, less AC's and space, etc. are required for a different work. In Cloud Computing, many handlers can access one local to recover and update their information without buying authorizations for variety of applications. The term "moving to Cloud" referred to as a company moving away from an outdated model of buy the devoted hardware and depreciate it concluded a certain amount of time to the latest trend of using a common Cloud organized structure and pay as one uses it.

Supporters privilege that Cloud Computing permits corporates to avoid unnecessary prices, and focus on missions that distinct their dealings instead of on organized structure. They also privilege that Cloud Computing provides organization to get their software running faster, with decent management and fewer maintenance, and permits Information Technology to extra fast adjust capitals to meet rise and fall the unexpected corporate demand. Cloud earners naturally use a "pay as you go" system. It leads to unpredictable high cost if managers do not adjust to the Cloud rating model. 
The current accessibility of high-database networks, lowprice pc's and storing devices as well as the wide approval of SOA, and usefulness Computing have leads to a development in public Cloud Computing. Cloud database suggestions an on-demand information subcontracting model, and is increasing status due to its springiness and less care and cost. Still, safety problem arises when information database is subcontracted to other Cloud service suppliers. It is necessary to allow Cloud customers to authenticate the veracity of their processed information, in case their information have been tactlessly sullied or any nastily conceded by unknown attacks.

One key issue in use of Cloud database is long-term archival, which gives a load that is carved once and hardly read. While the information is hardly read, it is essential to confirm its integrity for adversity retrieval or agreement with allowed requirements. Because it is usual to have a vast quantity of compressed information, full-file testing becomes expensive. Proof of information control have thus been projected to confirm the integrity of a huge file by inspecting only a segment of the file via many cryptographic primitives.

This method lasts to use casual hiding to give information secrecy during open reviewing, and influence guide hash tables to give fully lively actions on shared information. A process indicates an insert, delete or update task on a lone block in public information. CLOUD Computing has been taken as a fresh model of enterprise IT structure, which can form vast service of Computing, database and applications, and enable customers to get global, suitable and grid access to a shared configurable Computing assets with great proficiency and negligible economic price. Attracted by these features, both persons and enterprises are encouraged to upload their information to the Cloud, instead of buying software and hardware to succeed the information themselves.

In spite of the several rewards of Cloud services, subcontracting subtle data such as electronic mail, individual health archives, company investment data, administration forms, etc. to isolated servers carries some confidentiality anxieties. The Cloud amenity workers keep the info for handlers may admission delicate information without any approval. An all-purpose idea to defend the information privacy is to encrypt the information previously uploading. However, this determination reason a huge price in footings of statistics usability.

\section{LITERATURE SURVEY}

\section{OVERVIEW}

A work study is an explanation of pardon has been available on a matter by credited intellectuals and investigators. Infrequently you resolve be enquired to inscribe one as a isolated obligation, but extra regularly it is slice of the outline to a interpretation, enquiry or thesis. In writing the works appraisal, your resolve is to carry to your bibliophile what data and ideas have been reputable on a description, and what their optimistic and undesirable area[1],[2],[3]. As per a part of script, the work appraisal must be well-defined by a managerial idea (e.g., your study impartial, the matter you interchange or inscribe your belligerent thesis)

Also expanding your information round the area, inscription a work review occupancies you improvement and reveal services in two parts

1. INFORMATION SEEKING: the skill to image the works proficiently, using physical or electronic methods, to find a set of valuable tutelages and files

2. CRITICAL APPRAISAL: the skill to rub on philosophies of analysis to find impartial and legal studies.

Patient-centric model for health evidence exchanges the Personal health record (PHR) that becomes to be a growing[4][5][6]. It is usually outsourced to be kept at a third party, such as cloud suppliers. There have been long private concerns as personal health evidences could be known to several get-together servers and to unofficial gatherings. To promise the patients' controller over admission to their own PHR, it is a promising model to encrypt their data before subcontracting. Yet, several problems such as privacy disclosure scalability, risks in key management, elastic access and the user cancellation efficiency had continued to be the important tasks for attaining cryptographically and fine-grained enforced data access controller. It proposed a suite of mechanisms and the original patient-centric outline for records admission controller to PHRs deposited in semi-trusted headwaiters. To attain this scalable data and fine-grained starter controlling for PHRs. We influence attribute based encryption (ABE) methods to encrypt each patient's PHR record. Consider previous tasks in secure data subcontracting, this emphasis on proceeding the several data owner situation, and division the users passing the PHR organization into multiple safety provinces that critically decreases the key management complication for the users and proprietors.

A great graduation of patient private is guaranteed immediately by abusing multi expert ABE. This system allows active alteration of access policies or file points, thereby supports effectual on-demand attribute/user termination [7][8]. Extensive analytical and investigational results are obtainable which shows the scalability and efficiency of our projected outline.

Nowadays, encryption by searching is a major problem in cloud computing. The previous solutions mostly focused on search schemes based on arranged keyword, and nearly most of them rest on already defined keywords impassive in the module of directory assembly too inquiry. However, searches based on keyword based groups disregard the semantic symbol data of handlers' recovery and cannot totally competition workers' exploration meaning. So, in what way to plan a data-based search system and type semantic pursuit extra real in addition background-alert is a hard competition. This paper, proposed for the initial 
steps, we express and answer the difficulties of semantic pursuit placed on theoretical graphs (CGs) finished encoded agreement available information in obscuring calculating (SSCG)[9][10]. We initially service the skilled portion of "sentence scoring" in text summarization and trigon to expand the most significant and reduced problem verdicts from papers. We then translate these beginner's rulings into CGs. We plan a fresh way that can map CGs to tracks to perform predictable control of CGs[11][12]. Next, we list the repaid outcomes based on manuscript summarization notch. Still, they invent a rudimentary idea for SSCG and give a boldly enhanced scheme to satisfy the safety promise of searchable symmetric encryption (SSE). Finally, we took a real-world dataset called the $\mathrm{CNN}$ dataset to quiz our deal. The fallouts found from the project describes the benefit of our system.

Here, they addresses the artifice of confidentiality preservative vicarious term hunt in the cloud. It considers a state where an evidence proprietor subcontracts their statistics to a cloud server and gives the examine competences to a group of third party users. In the view of partial-veracious cloud servers, the holder of data does not require to reveal any info about the outsourced data. Yet it still wants to ameliorate from a high similar cloud situation. Furthermore, the data holder needs to be safeguarded which assigns the examine functionality to other parties. It did not sanction other parties to expose the privacy of the outsourced data, whether it does avert the data holder from professionally withdrawing the approbation of those sanctioned parties. Hence, they recommend a word quest protocol that shapes methods of keyed hash purposes, insensible pseudo-arbitrary purposes and Cuckoo hashing to construct identifiable catalogue for the subcontracted facts, and uses remote info recovery of small info to ensure that term exploration queries does not disclose any info about the data to the cloud provider. We cumulate attribute-predicated encryption and insensate pseudo-arbitrary tasks to procure an effectual cancellation of sanctioned third parties. It is felicitous for the cloud as it can be facilely optimized.

Over cloud server, Active searchable encryption allows data owner to store the active collection of encrypted files to the cloud server and make search tokens of queries. Upon receiving a token, the server can achieve those examine on the encrypted data while conserving privacy. Unlike many previous works that dedicated on a singleuser scheme, we present the active searchable encryption preparation with multi-user privacy search for cloud computing. For access the data, we consider the use scenario of cloud storage services where an organization subcontracts its data to the cloud and approves a collection of users. Our technique is dependent on a red-black data structure which is highly parallelizable and energetic, and its security is proven in the unplanned oracle replica.

\section{EXISTING SYSTEM}

An incipient enterprise IT architecture has been emerged by Cloud computing. Private care has perpetuated a primary obstacle eschewing the acceptance of cloud computing by a more sizably voluminous variety of users/applications. If critical information are subcontracted to cloud, then owners of data were not artificially become worried with the confidentiality of their data in the cloud and outside. The encrypted information can be effectually exploited then becomes another fresh task. Symmetric cryptography established methods are limpidly not fit for this scenery due to the tall difficulty of stealthy word organization. Sanctioned keyword find might be understood in one-owner setting by ostensibly describing a server-enforced utilizer list which takes the accountability to controller sincere user hunt proficiencies. Examination can only be approved by the server with the help of genuine user harmonizing keys on the utilizer list. Within a dataset, those methods doesn't understand fine-grained owner-enforced finding approbation and thus incapable to distribute distinguished access sanctions for individual users. Asymmetric cryptography is well matched to this dynamic scenery by encrypting individual influence with dissimilar general keys. Attribute-predicated keyword find method with effective utilizer reversal (ABKS-UR). Users can engender their own exploration abilities without relying on an always connected trustworthy ascendancy.

\section{IMPLEMENTATION}

Our proposed method is moderately established on the opinion that, in the real-world application situations each facts file could be connected with a group of characteristics that are substantial in the viewpoint of curiosity. The user is permitted to access, the admittance construction of every user can be defined to be sole reasonable manifestation over those characteristics to replicate the choice of data files. The reasonable appearance can characterize any wanted data set by finegraininess of data admittance controller is accomplished. We declare a public word contents for each characteristic to enforce the access infrastructure. Data file sets are encrypted by public word constituent responsible to their characteristics. User secret passwords are declared to reproduce their permission arrangements so the user is capable to decrypt a cipher text if the data file characteristics contented person's permission infrastructure.

A model gets about the efficacy profit, as related to former tasks, in this difficulty of encryption has correlated the amount of characteristics connected to the data file. It is liberated to the amount of workers in the system and information file deletion or creation and new user donation processes just disturb current user/file without connecting system long data file keying or update. One enormously inspiring issue with this model is the application of user cancellation, which would unavoidably need re-encryption of data files nearby to the leaving user, and may need update of secret passwords for all the outstanding users. If these responsibilities are achieved by data proprietor, it would present a heavy calculation upstairs and may also 
require the records owner to be continuously connected. We attain this objective by abusing and exclusively joining methods of Key Policy attribute-based encode (KP-ABE) and Flap encode. In cloud computing, it has striking possessions of manager secret key responsibility and accomplishes scalability, fine-graininess and data privacy for data admittance controller.

\section{CONCLUSION}

We realize this goalmouth by developing and exclusively merging the systems of key strategy attribute-based encryption (ABE) and idle flap-encryption. Our future system also has relevant belongings of operator access pleasure privacy and user stealthy key answerability and bring to an end fine-graininess, scalability and information privacy for information entree governor in cloud computing. Wide analysis displays that our future arrangement is very well-organized and provably locks under present safety models.

\section{REFERENCES}

[1] S.Saravanan, Arivarasan."An efficient ranked keyword search for effective utilization of outsourced cloud data" Journal of Global Research in Computer Science, Vol4(4), pp:8-12

[2] W. Sun, S. Yu, W. Lou, Y. T. Hou, and H. Li, "Protecting Your Right: Attribute-based Keyword Search with Fine-grained Ownerenforced Search Authorization in the Cloud," in IEEE INFOCOM, pp. 226-234, 2014.

[3] S. Yu, C. Wang, K. Ren, and W. Lou, "Achieving secure, scalable,and fine-grained data access control in cloud computing," in Proc.of IEEE INFOCOM, pp. 1-9, 2010.

[4] M. Li, S. Yu, Y. Zheng, K. Ren, and W. Lou, "Scalable and secure sharing of personal health records in cloud computing using attribute-based encryption," IEEE TPDS, vol. 24 , no. 1, pp. 131143, 2013.

[5] S. Kamara and K. Lauter, "Cryptographic cloud storage," in Financial Cryptography and Data Security, pp. 136-149, 2010.

[6] D. Song, D. Wagner, and A. Perrig, "Practical techniques for searches on encrypted data," in Proc. of IEEE S\&P, pp. 44-55, 2000.

[7] S Saravanan, V Venkatachalam , Improving map reduce task scheduling and micro-partitioning mechanism for mobile cloud multimedia services" International Journal of Advanced Intelligence Paradigms, Vol 8(2),pp157- 167,2016.

[8] S Saravanan, V Venkatachalam "“ Advance Map Reduce Task Scheduling algorithm using mobile cloud multimedia services architecture" IEEE Digital Explore,pp21-25,2014.

[9] S.Swathi "Preemptive Virtual Machine Scheduling Using CLOUDSIM Tool", International Journal of Advances in Engineering, 2015, 1(3), 323 -327 ISSN: 2394-9260, pp:323-327.

[10] S Saravanan, V Venkatachalam, S Then Malligai "Optimization of SLA violation in cloud computing using artificial bee colony"2015, 1(3), 323 -327 ISSN: 2394-9260, pp:410-414.

[11] S. Saravanan, Vikram R ,"Improved Performance Analysis Image Segmentation Based on Cluster Image", Journal of Chemical and Pharmaceutical Sciences,issue 1,2017,pp92-95

[12] S. Saravanan, Vikram R ," Evolutionary Calculations on Gravitational Interactions Method of Global Leader Organize ", Journal of Chemical and Pharmaceutical Sciences, issue 1,2017,pp115-118 